\title{
Schwinger-boson mean-field theory for $S=1$ open spin chains
}

\author{
T. K. Ng* \\ AT\& T Bell Laboratories, Murray Hill, New Jersey 07974
}

(Received 16 October 1991)

\begin{abstract}
The properties of $S=1$ open spin chains is studied using Schwinger-boson mean-field theory. It is found that localized $S=\frac{1}{2}$ states exist at the ends of chains and that the ground states of chains with even and odd number of sites are fundamentally different. Properties of the localized end states are discussed.
\end{abstract}

In the past few years there has been increasing interest in the study of one-dimensional spin chains with nearestneighbor antiferromagnetic exchange interaction $J$, following the conjecture by Haldane ${ }^{\prime}$ that integer spin chains have a singlet ground state with a finite gap $E_{H}$ in the excitation spectrum, whereas the excitation spectrum for half-integer spins is gapless. More recently, the physics of $S=1$ open spin chains has received new attention stimulated by the discovery of fractional $S=\frac{1}{2}$ spin states localized at the ends of the chain. ${ }^{2.3}$ It is generally believed that the qualitative behavior of the $S=1$ spin chain can be understood in terms of the valence-bond-solid (VBS) model ${ }^{4,5}$ where $S=1$ spin on each site is represented by symmetrization of two $S=\frac{1}{2}$ spins and a manybody singlet ground-state wave function is formed by making two valence bonds ${ }^{4,5}$ from each site to adjacent neighbors. It has been shown rigorously ${ }^{5}$ that the correlation function decays exponentially in the VBS state and that there exists an energy gap in the excitation spectrum. For an open chain, unpaired bonds are left at each end of the chain, corresponding to effective localized $S=\frac{1}{2}$ objects. A similar picture for the ground state of the Heisenberg model is believed to be valid except that the valence bonds are not restricted to form only between nearest-neighbor sites ${ }^{6}$ and a residual effective interaction $\sim J e^{-L / \xi}$ ( $L$ is the length of chain, $\xi$ is the correlation length) between the two end states is believed to exist. The residue interaction is believed to be antiferromagnetic for chains with an even number of sites and ferromagnetic for chains with an odd number of sites, resulting in a $S=0$ singlet ground state for even chains and $S=1$ triplet ground state for odd chains. ${ }^{7}$ This physical picture has been confirmed by exact diagonalization on open chains up to 14 sites. $^{8}$

In this paper we shall study open $S=1$ Heisenberg spin chains using the Schwinger-boson mean-field theory (SBMFT) proposed by Arovas and Auerbach ${ }^{9}$ for the Heisenberg model. The theory has been applied successfully to infinite Heisenberg spin systems in both one- and two-dimensions. ${ }^{9,10}$ (In the one-dimensional case, the theory works well only with integer spin chains, presumably due to the neglect of topogical excitations in the SBMFT. ${ }^{9}$ ) The theory is the leading term in a systematic $1 / N$ expansion and has the advantage that computations can be made easily on long chains. It also provides a simple physical picture for understanding the properties of the $S=\frac{1}{2}$ end states. We shall study chains with various lengths and compare the mean-field results with theoretical expectations and experimental results. Effects of fluctuations beyond mean-field theory and various asymmetric terms in the Hamiltonian ${ }^{3}$ will also be discussed.

To begin with, we first briefly review properties of infinite Heisenberg spin chains in the SBMFT. ${ }^{9}$ The SBMFT can be interpreted as a variational approach with VBS-type trial ground-state wave functions, except that (1) valence bonds joining arbitrary sites on opposite sublattices are allowed ${ }^{6,11}$ and (2) the constraint that there are two spin- $\frac{1}{2}$ objects on each site is relaxed and is satisfied only on average. "I The probability distribution of the valence bonds is determined variationally in the SBMFT. The major source of error in the theory is believed to be the relaxation of constraint ${ }^{9.10}$ which results in quantitatively unreliable results for properties like ground-state energy and correlation length. Elementary excitations in the theory are $S=\frac{1}{2}$ spinons with a gap $m$ in the excitation spectrum determined self-consistently by the mean-field equations. Notice the physical excitations have $S=1$ and are composed of pairs of spinons resulting in gap $E_{H}=2 m$ in the physical excitation spectrum. The value of the Haldane gap $E_{H}$ and correlation length $\xi$ are $E_{H} \sim 0.17 \mathrm{~J}$ and $\xi \sim 12.2$ lattice spacings, respectively, in the SBMFT, whereas the best available results have $E_{H} \sim 0.41 J$ and $\xi \sim 6.2$, indicating that SBMFT can be trusted only qualitatively.

The details of the SBMFT have been worked out by Arovas and Auerbach ${ }^{9}$ and we shall not repeat them here. The resulting mean-field Hamiltonian for a chain of $N$ sites is

$$
H_{\mathrm{MF}}=-J \sum_{i=1, \ldots, N-1}\left[\Delta_{i, i+1}\left(\bar{Z}_{i \uparrow} \bar{Z}_{i+1 \downarrow}-\bar{Z}_{i \uparrow} \bar{Z}_{i+1 \uparrow}\right)+\text { c.c. }-\left|\Delta_{i, i+1}\right|^{2}\right]+\sum_{i=1, \ldots, N} \lambda_{i}\left(\bar{Z}_{i \uparrow} Z_{i \uparrow}+\bar{Z}_{i \downarrow} Z_{i \uparrow}-2 S\right),
$$

where $\bar{Z}_{i \sigma}\left(Z_{i \sigma}\right)$ is a Schwinger boson operator creating (annihilating) a spin $\sigma\left(\sigma= \pm \frac{1}{2}\right)$ boson on site $i, S=1$ is the spin magnitude, and $\Delta_{i, i+1}$ and $\lambda_{i}$ are mean-field parameters determined by the self-consistent equations

$$
\Delta_{i, i+1}=\left\langle Z_{i \uparrow} Z_{i+1 \downarrow}-Z_{i \downarrow} Z_{i+1 \uparrow}\right\rangle, i=1, \ldots, N-1
$$


and

$$
\left\langle\bar{Z}_{i \mid} Z_{i \uparrow}\right\rangle+\left\langle\bar{Z}_{i \mid} Z_{i \downarrow}\right\rangle=2 S, i=1, \ldots, N .
$$

Notice that Eq. (2b) implies that the constraint that there are two bosons per site is satisfied only on average.

For infinite system $N \rightarrow \infty$ and with the periodic boundary condition $\Delta_{i, i+1} \rightarrow \Delta, \lambda_{i} \rightarrow \lambda$ become constants because of translational invariance. However, for finite, open systems $\Delta_{i, i+1}$ and $\lambda_{i}$ are different on every site $i$ and have to be determined self-consistently for every site. We shall first consider the situation where there are even number of sites $N=2 M$ and the ground state is believed to be a singlet. It is convenient to divide the sites into $A$ and $B$ sublattices and the mean-field Hamiltonian (1) can be diagonalized by a Bogoliubov-de Gennes transformation

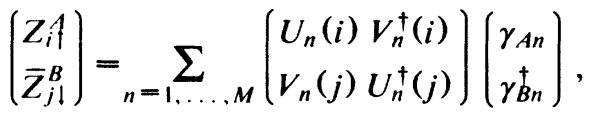

where $i$ and $j$ runs from 1 to $M$ denotes sites on opposite sublattices. In this notation $U_{n}^{\dagger}(i)=U_{n}^{*}(M-i)$ and $V_{n}^{\dagger}(i)=V_{n}^{*}(M-i)$. A similar equation is also obtained for the pairs $\left(\bar{Z}_{i \downarrow}^{A}, Z_{j \dagger}^{B}\right)$. $\left(U_{n}, V_{n}\right)$ are eigenstates of the eigenvalue equation

$$
\begin{aligned}
& \lambda_{i} U_{n}(i)-\sum_{j^{\prime}=i \pm 1} \Delta_{i j^{\prime}} V_{n}\left(j^{\prime}\right)=\varepsilon_{n} U_{n}(i), \\
& \lambda_{j} V_{n}(j)-\sum_{i^{\prime}=j \pm 1} \Delta_{i^{\prime} j}^{*} U_{n}\left(i^{\prime}\right)=-\varepsilon_{n} V_{n}(j),
\end{aligned}
$$

where $\varepsilon_{n}>0$ are the corresponding eigenvalues and the eigenvectors $\left(U_{n}, V_{n}\right)$ satisfy the orthonomality condition

$\sum_{i=1, \ldots, M} U_{n}^{*}(i) U_{m}(i)-\sum_{j=1, \ldots, M} V_{n}^{*}(j) V_{m}(j)=\delta_{n m}$.

$\left(U_{n}, V_{n}\right)$ can be interpreted as the wave function of an excited $S=\frac{1}{2}$ spinon state with energy $\varepsilon_{n}$. It can be shown that the magnetization carried by a single spinon state $n$ is $m_{i} \propto\left|U_{n}(i)\right|^{2}$ on sublattice $A$ and $m_{j}$ $\propto-\left|V_{n}(j)\right|^{2}$ on sublattice $B$. Thus a spinon carries staggered magnetization. The orthonomality condition (5) implies that although the absolute value of magnetization carried by a spinon can be large on each site, the total magnetization after summing up contributions from the two sublattices is exactly equal to $\frac{1}{2}$. In the SBMFT, the ground state of the spin chain consists of condensates of pairs of spinons with opposite extra magnetization localized on opposite sublattices and with a free-spinon excitation spectrum

$$
H_{\mathrm{MF}}=\sum_{n, \sigma} \varepsilon_{n}\left(\gamma_{A n \sigma}^{\dagger} \gamma_{A n \sigma}+\gamma_{B n \sigma}^{\dagger} \gamma_{B n \sigma}-2\right),
$$

where $\gamma_{A \sigma}^{\dagger}\left(\gamma_{B \sigma}^{\dagger}\right)$ creates a spin $\sigma$ spinon with extra magnetization localized in sublattice $A(B)$ and with groundstate energy $-2 \sum_{n, \sigma} \varepsilon_{n}$.

We have solved the mean-field equations (3)-(5) numerically for even chains at zero temperature from 12 to 100 sites and find that localized spinon states with $\varepsilon_{L}<m$ at the ends of the chain exists for all chains we considered. Before going to the numerical results we shall first give a qualitative explanation why localized states are found at the ends of the chain in the SBMFT. Let us first consider an infinite chain. In this case, $\Delta_{i, i+1}$ and $\lambda_{i}$ are constants in Eq. (1) and the spinon wave function $\left(U_{n}, V_{n}\right)$ are plane waves because of translational invariance. For a finite chain, it is expected that plane waves will get scattered back from the ends of chain resulting in formation of standing waves with nodes at the ends of chain. As a result, the amplitude of the spinon wave functions will be much reduced at the ends of chain and so does the magnitude of spin at the ground state since the ground state is just a condensate of spinon pairs in the SBMFT. Howev$\mathrm{er}$, this is impossible because of the constraint that there must be two Schwinger bosons on every site on average. To satisfy the constraint a localized state is generated at each end of chain self-consistently in the SBMFT. (Mathematically, the magnitude of $\lambda$ at the ends are reduced to create potential wells for spins.)

Figure 1 shows the low-energy part of our calculated spinon dispersion $\varepsilon_{n}$ for a 64-site chain (denoted by crosses). The solid line is the energy spectrum for the corresponding infinite chain. The spinon excitation spectrum is fourfold degenerate corresponding to spinons with opposite spin and with extra magnetization localized on opposite sublattices. We have shown only two degenerate sets of states in Fig. 1, corresponding to spinons on opposite sublattices. Notice that this is not a physical excitation spectrum which has $S=1$ and corresponds only to gauge invariant objects in SBMFT. ${ }^{10}$ Notice also that there is only one state with $\varepsilon_{l}<m$ at each sublattice, in agreement with the VBS model that only one unpaired spin exists in each end of spin. Similar behavior is also found for all other chains with different lengths, except that the energy $\varepsilon_{L}$ varies. The dependence of $\varepsilon_{L}$ on the length of chain $N$ is plotted in Fig. 2 on a semilog scale (denoted by crosses). It is apparent that $\ln \varepsilon_{L} \propto N$ for large $N$, in agreement with naive expectation. However, the decaying length $l\left(\varepsilon_{L} \sim e^{-l / l}\right)$ found by fitting the data is $l \sim 28$, which is about 2.3 times larger than the mean-field correlation length $\xi \sim 12.2$. The asymptotic behavior $\ln \varepsilon_{L} \sim N$

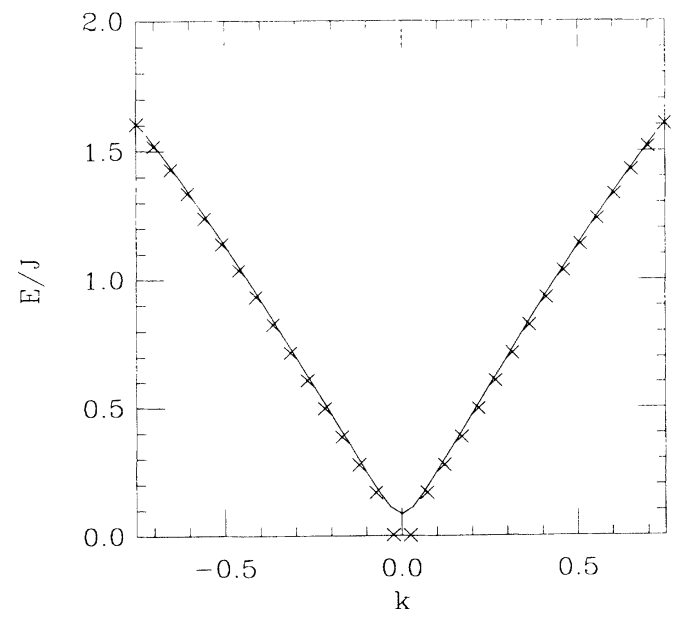

FIG. 1. Low-energy part of the spinon excitation spectrum for a 64-site open chain (crosses). The solid line is the infinite chain excitation spectrum. Notice the existence of two states with energy $\varepsilon_{l}<m$ in the open chain. 


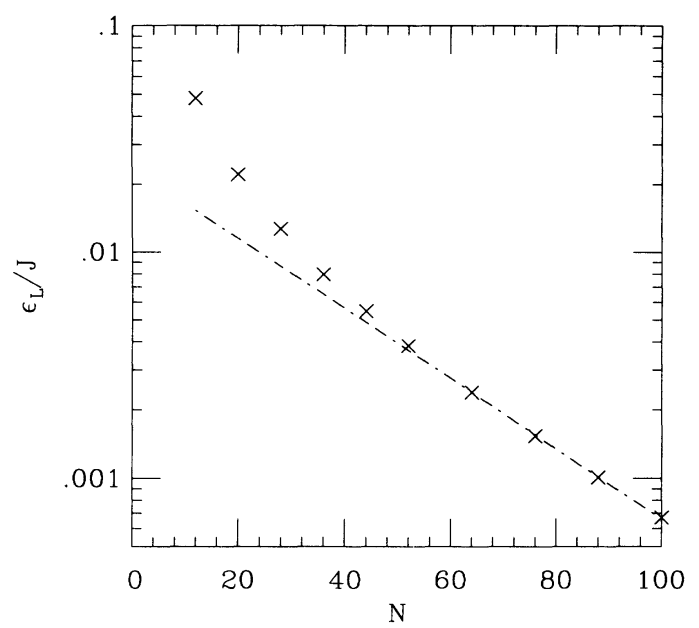

FIG. 2. The energy of the end-state spinon $\varepsilon_{l}$, as a function of chain length $N$ on a semilog scale. The straight dashed line is the fit by $\varepsilon_{l}=0.235 J \exp (-N / 28)$.

is found only for $N>50$, also in agreement with $l \geq 2 \xi$. (Notice that there are two end states in one chain. Thus asymptotic behavior is obtained only for $N \geq 2 l \sim 56$.)

The large decaying length $l$ has a simple explanation in SBMFT. The reason is that the correlation length $\xi$ is defined for a physical $S=1$ excitation which involves two spinons with excitation gap $E_{H}=2 m$ whereas a single $S=\frac{1}{2}$ excitation has gap $m$. Thus the end states have decaying length $-2 \xi$ instead of $\xi$ because of the half gap in the single spinon excitation spectrum. Notice that this argument is independent of SBMFT and depends only on the "fractionalized" spin quantum number of the end states. Thus although the SBMFT can be trusted only qualitatively, we believed that the result $l \sim 2 \xi$ is indeed a valid prediction. (Notice, however that a counter argument to this result is that in infinite chains, it is known that the $S=\frac{1}{2}$ spinons are confined and the real elementary excitations has $S=1$. Thus, only $\xi$ can be the physical length scale, not $2 \xi$.)

Now let us consider chains with an odd number of sites where the ground state is believed to be a $S=1$ triplet. It is less straightforward to apply SBMFT in this situation because SBMFT corresponds to $S=0$ singlet wave functions. To overcome this difficulty we consider even chains with $N=2(2 M+1)$ sites ( $M$ is an integer) and gradually reduce the coupling $J_{m}$ at the middle of the chain. In the limit $J_{m} \rightarrow 0$, we get two identical odd chains. For finite $J_{m}$ much greater than the energy of the end states $\varepsilon_{L}$, it is expected that the $S=1$ end states at the two odd chains will be weakly coupled by $J_{m}$, resulting in a singlet ground state with excitation energy $\varepsilon_{c} \sim J_{m}$ for the coupled chains. We have performed the above computation and the resulting spinon excitation spectrum for a $2 \times 13$ chain is shown in Fig. 3 (denoted by crosses), with $J_{m} \sim 0.01 J$. We have shown only 13 states, corresponding to spinon states on one of the coupled chains. The states to the left (right) of the center of the figure correspond to spinon states with extra magnetization on the $A(B)$ sublattice. Notice that there is one more spinon state on the $A$ sublattice. For comparison we have also plotted the spinon spec-

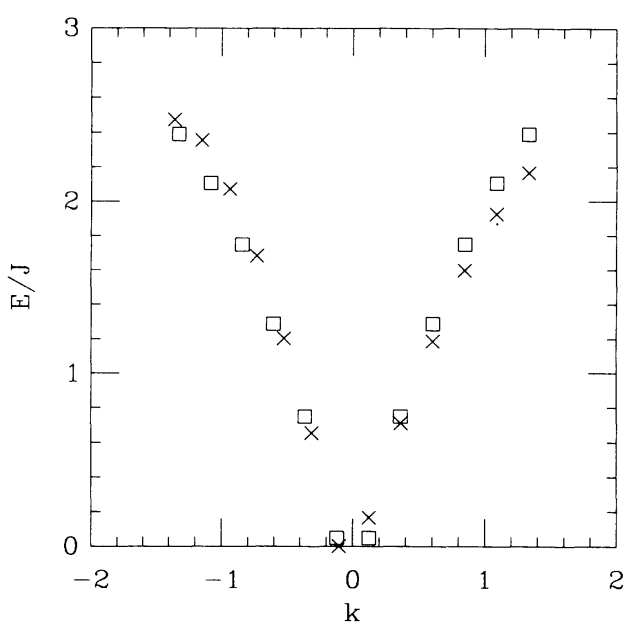

FIG. 3. Spinon excitation spectrum for two weakly coupled chains $\left(J_{m} \sim 0.01 J\right)$ with $2 \times 13$ sites (crosses). The excitation spectrum for an even chain with 12 sites (squares) is also shown for comparison.

trum for a chain with 12 sites (denoted by squares). It is clear that the two excitation spectra look rather different. Besides having one more unpaired spinon state, the degenerate pairs in the 12-site chain are split in the 13-site case. Notice that in the limit $N \rightarrow \infty$, the effect of asymmetry between the $A$ and $B$ sublattices should vanish and the splitting between the two paired states should go to zero. To examine whether the ground state is a triplet, we have examined the properties of the spinon spectrum as a function of $J_{m}$. For states other than the lowest one, we find that changing $J_{m}$ from $\sim 0.02$ to 0.005 does not change the spectrum in any significant way. However, for the lowest rate state $\varepsilon_{1}$ we find that $\varepsilon_{1} \sim 0.15 J_{m}$ to a good approximation, in agreement with our expectation that the two coupled chains have excitation energy $\varepsilon_{c} \sim J_{m}$. Notice that the second lowest state $\varepsilon_{2}$ also has a much lower energy in comparison with other states. In fact, it is expected that in the limit $N \rightarrow \infty, \varepsilon_{2} \sim e^{-L / l} \rightarrow 0$ which together with state $\varepsilon_{1}$ corresponds to decoupled $S=\frac{1}{2}$ spinon states localized at the ends of the chain, as in the even chain case. To test these expectations, we have performed a similar calculation for a 46-site system with reduced $J_{m}$ and find that indeed the splitting between the spinon states on the two sublattices are reduced and $\varepsilon_{2}$ has a much reduced energy (smaller than $m$ ). Unfortunately, because of the smallest of $\varepsilon_{L}$ for the 46 -site chain, it becomes numerically difficult to reduce $J_{m}$ to $J_{m}<\varepsilon_{L}$, and the detailed behavior of the two lowest states $\varepsilon_{1}, \varepsilon_{2}$ were not obtained in this regime. We have, however, studied chains shorter than 26 sites and find that $\varepsilon_{1} \sim J_{m}$ for small enough $J_{m}$ in all cases, indicating that the ground states for odd chains and even chains are indeed fundamentally different.

We shall now study the behavior of the localized endstate spinon wave function. The results are extracted from the even chain calculations where much longer chains can be examined.

As is explained above, spinons behave as free $S=\frac{1}{2}$ objects in the SBMFT and the wave function of a spinon 
represents staggered magnetization with opposite sign on the two sublattices and with total extra magnetization $\frac{1}{2}$. For the end state, it is expected that the spinon is exponentially localized at the ends of chain with decaying length $l \sim 2 \xi$. This is indeed found numerically in our calculation except that since we are working at zero temperature, a tunneling effect of the spinon from one end to other always exists and exponential decay of the wave function as distance is found only for distance $D<N / 2$. For $D>N / 2$, the amplitude of the spinon wave function increases again. For $D<N / 2$, except for the first few sites, the magnetization carried by the end state spinon can be fitted well by the form $m\left(x_{i}\right)=(-1)^{i} f\left(x_{i}\right)$, where $f(x)$ is a smooth, exponentially decaying function with decaying length $l / 2 \sim 14$, since $m \propto$ (wave function) ${ }^{2}$. The absolute magnitude of the magnetization is found to be large on the first few sites, with $|m| \sim 0.7(>1 / 2)$ on the first site. This large magnetization can be understood in a simple way as follows. Assuming that the absolute magnitude of magnetization $f\left(x_{i}\right)$ is smooth everywhere, the total magnetization carried by a spinon is thus

$$
\begin{aligned}
M & =\sum_{i}(-1)^{i} f\left(x_{i}\right) \sim-\frac{1}{2} \int_{0}^{\infty} f^{\prime}(x) d x \\
& =\frac{1}{2}[f(0)-f(\infty)]=\frac{1}{2} .
\end{aligned}
$$

With $f(\infty) \rightarrow 0$, we find $f(0)=1$, i.e., a very large magnetization is expected to exist on the first site. This large magnetization is actually reduced in the SBMFT, because $f(x)$ is not "smooth" on the first few sites. Notice that this argument is independent of SBMFT and depends only on the smoothness of $f(x)$ far away from the ends. Thus we believe that although the absolute values of magnetization we find in the SBMFT is probably incorrect, they are likely to be rather large (i.e., of order 1) in the "correct" theory. Notice that this and the spinon wave-function decaying length $l \sim 2 \xi$ can be tested in neutron experiments or can be tested against exact diagonalization results on $S=1$ open chains. Unfortunately, a rather long chain $(N \gtrsim 4 \xi \sim 25)$ is needed to test the $l \sim 2 \xi$ result whereas the best available calculation at present is only up to 14 sites. $^{8}$

There are two main drawbacks to the SBMFT. First of all, the constraint that there are two Schwinger bosons $(S=1)$ per site is only satisfied on average. Second, interaction between spinons are neglected. In the infinite chain case, it is known that the correct dynamics for the system can be recovered only after taking into account interaction between spinons ${ }^{12}$ and, correspondingly, the static properties of the system are renormalized. ${ }^{12}$ In the present case, because of the existence of gap $E_{H}$ in the excitation spectrum, we believe that the dynamics of the end-state spinons would not be modified qualitatively (it cannot decay into other states) at least for temperature $T \ll E_{H}$ and the main effect of interaction would be the renormalization of the $g$ factor characterizing the response of the end-state spinons towards external perturbation.

Experimentally it appears that asymmetric $g$ factor $\left(g_{x} \neq g_{y} \neq g_{z}\right)$ are found in open $S=1\left[\mathrm{Ni}\left(\mathrm{C}_{2} \mathrm{H}_{8} \mathrm{~N}_{2}\right)_{2-}\right.$ $\left.\left(\mathrm{NO}_{2}\right)\right]_{\mathrm{ClO}_{4}}$ chains ${ }^{3}$ because of asymmetry in the underlying Hamiltonian

$$
H \sim \sum_{\substack{\langle i, j\rangle \\=x, y, z}} J_{\mu} S_{i \mu} S_{j \mu}+\sum_{i, \mu} D_{\mu}\left(S_{i \mu}\right)^{2},
$$

where $J_{\mu}, D_{\mu}$ are slightly different in different directions. An asymmetric $g$ factor is absent in SBMFT but can be understood qualitatively by considering a modified meanfield theory with additional decoupling $\langle S S\rangle \sim\langle S\rangle\langle S\rangle$ in the presence of magnetic field. The response of the localized end-state spinon towards magnetic field would be renormalized by a Stoner (random-phase approximation) type factor which is different for different directions. Physically, the inclusion of interaction terms take into account the effect that not only a spinon is excited at the presence of external field, but its wave function is also modified, leading to a renormalized $g$ factor.

The author thanks B. S. Shastry and S. Geschwind for many helpful discussions.
* Present address: Department of Physics, Hong Kong University of Science and Technology, Clear Water Bay Road, Kowloon, Hong Kong.

'F. D. M. Haldane, Phys. Rev. Lett. 50, 1153 (1983); J. Appl. Phys. 57, 3359 (1983).

${ }^{2}$ M. Hagiwara, K. Katsumata, I. Affleck, B. I. Halperin, and J. P. Renard, Phys. Rev. Lett. 65, 3181 (1990).

${ }^{3}$ S. H. Glarum, S. Geschwind, K. M. Lee, M. L. Kaplan, and J. Michael, Phys. Rev. Lett. 67, 1614 (1991).

${ }^{4}$ I. Affleck, T. Kennedy, E. H. Lieb, and H. Tasaki, Phys. Rev. Lett. 59, 799 (1987).
${ }^{5}$ J. Affleck, T. Kennedy, E. H. Lieb, and H. Tasaki, Commun. Math. Phys. 115, 477 (1988).

6S. Liang, Phys. Rev. Lett. 64, 1597 (1990).

${ }^{7}$ E. H. Lieb and D. C. Mattis, J. Math. Phys. 3, 747 (1962).

${ }^{8}$ T. Kennedy, J. Phys. Condens. Matter 3, 5757 (1990).

${ }^{9}$ D. P. Arovas and A. Auerbach, Phys. Rev. B 38, 316 (1988).

${ }^{10}$ A. Auerbach and D. P. Arovas, Phys. Rev. Lett. 61, 617 (1988).

I'C. L. Kane, P. A. Lee, T. K. Ng, B. Chakraborty, and N. Read, Phys. Rev. B 41, 2653 (1990).

${ }^{12} \mathrm{~A}$. Chubukov (unpublished). 